\title{
Throughput Maximization on the Downlink of a CDMA System
}

\author{
M. Kemal Karakayali Roy Yates Leo Razoumov \\ Department of Electrical and Computer Engineering \\ Wireless Information Networks Laboratory (WINLAB) \\ Rutgers University, 73 Brett Road Piscataway, NJ 08854-8060
}

\begin{abstract}
We propose a rate scheduling algorithm to maximize the network throughput of a variable data rate CDMA system and prove its optimality. The system uses OVSF (orthogonal variable spreading factor) codes and the algorithm finds the optimum rate assignments on the binary code tree under constraints on the total transmit power and minimum QoS (rate) requirement of each user. The algorithm is optimal in the sense that it maximizes the total network throughput within the constraints and achieves this with minimum possible power. The algorithm works in a greedy fashion and has a polynomial time complexity of $O(N)$, where $N$ is the number of users.

We also extend our results to a more general set of combinatorial optimization problems where the user rates can be any integer multiples of a basic rate (such as Multi-Code CDMA), not necessarily the set of rates on the binary tree structure, but the optimal solutions are still greedy achievable.
\end{abstract}

\section{INTRODUCTION}

Unlike voice-based second generation cellular networks, third and fourth generation mobile networks will provide multimedia services with variable data rates and different service classes in addition to classical voice service. In the third generation W-CDMA standard, variable data rate service is provided by assigning each user a single spreading code with variable length [1]. The set of spreading codes used in such a scheme is obtained from a binary tree structure (Figure 1) and called Orthogonal Variable Spreading Factor (OVSF) codes [2]. On the other hand, in Multi-Code CDMA systems, each user can be provided with multiple spreading codes of fixed length, depending on the users' rate requests.

Recent studies on variable data rate CDMA systems focus on efficiency of dynamic spreading code assignment schemes, especially for the systems employing OVSF codes [3], [4], [5], [6]. The basic question these studies attempt to answer is how to accomodate an incoming user's rate request on the OVSF code tree. For a Multi-Code CDMA system it is easy to handle an incoming user's request, if the requested rate is achievable within available network bandwidth, as many as the number of spreading codes corresponding to the requested amount is assigned to the incoming user. Since all fixed length spreading codes are mutually orthogonal in Multi-Code systems, assigning an unused spreading code to a new user does not affect the existing users. On the other hand inherent binary tree structure of OVSF codes in variable spreading CDMA systems complicates the code management issue. As an example in Figure 1, assume there are 2 existing users in the system and their spreading codes are located at $C_{2,1}$ and $C_{2,3}$ on the binary code tree. Thus the total used system bandwidth is $R / 2$ and half of the the total bandwidth is still unused. In this case, if a new user requests $R / 2$, the system bandwidth is available for this request however the system cannot locate a spreading code for the new user since $C_{1,1}\left(C_{1,2}\right)$ is not orthogonal to the existing code $C_{2,3}$ $\left(C_{2,1}\right)$. This phenomena is known as code blocking [3].

In [3], [5] and [6], dynamic code assignment schemes are proposed to minimize the code blocking probability and to minimize the number of existing spreading codes relocated in case of an incoming user. In [4], the authors propose a protocol which uses a credit-based reservaton scheme to prioritize users and attempts to provide fairness to each user while providing per-connection bandwidth gurantee to bursty data applications. However none of the above studies question throughput limiting system resources other than the total available bandwidth, such as the total transmit power.

In this study we question variable data rate CDMA system from a radio resource management point of view. For both Multi-Code and variable spreading CDMA systems, our objective is to identify the maximum achievable network throughput given available network resources (both power and bandwidth), and to find a computationally simple (polynomial time if possible) rate assignment algorithm resulting in the set of rates achieving the maximum throughput. In addition for variable spreading systems, we show that the set of optimum rates concluded by our algorithm are always realizable on the binary code tree as a result of the Kraft Inequality (mentioned later in the paper), the way the spreading code replacements or shifts occur during each step of our algorithm is a subject of [3], [5] and [6] and not adressed in this paper.

Figure 1 shows how OVSF codes are obtained from a binary tree structure. Note in the figure that $C_{i, j}$ represents $j$ th node on layer $i$ on the binary tree and it corresponds to a unique signature sequence of length $2^{i}$ and rate $R_{0} / 2^{i}$ where $R_{0}$ is the root rate corresponding to the node $C_{0,1}$, also note that all the nodes on the same layer have the same spreading factor. Moreover, orthogonality of the assigned signature sequences is guaranteed by the fact that none of the parent-child node pairs is assigned to different users at the same time. As an example, the nodes $C_{1,2}$ and $C_{2,4}$ in figure 1 cannot be in use at the same time since $C_{1,2}$ is a prefix of $C_{2,4}$. Accordingly, the resulting set of assigned signature sequences must have the prefix-free property. 


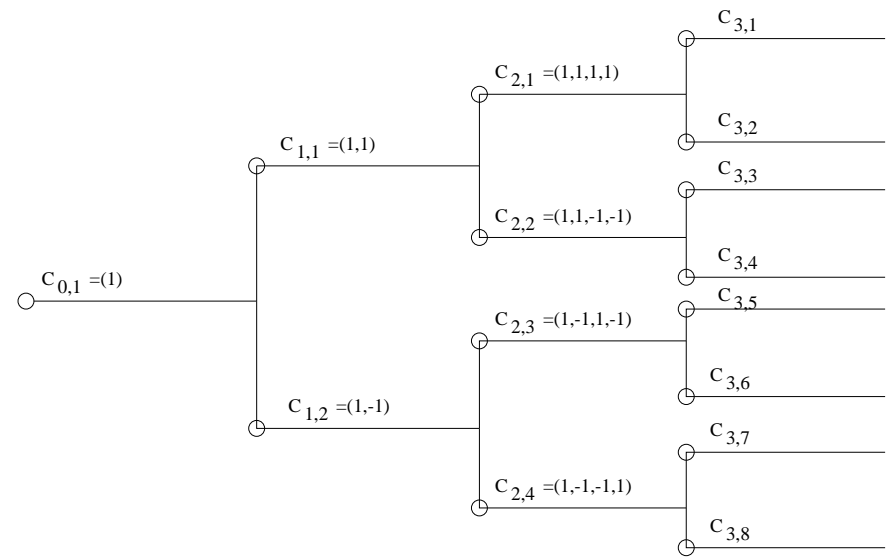

$\begin{array}{llcc}\text { layer } 0 & \text { layer } 1 & \text { layer } 2 & \text { layer } 3 \\ \mathrm{SF}=1 & \mathrm{SF}=2 & \mathrm{SF}=4 & \mathrm{SF}=8 \\ \text { Rate }=\mathrm{R} & \mathrm{Rate}=\mathrm{R} / 2 & \mathrm{Rate}=\mathrm{R} / 4 & \text { Rate=R/8 }\end{array}$

Fig. 1. OVSF Code Tree. $C_{i, j}$ represents node $j$ on layer $i$ and has a length of $\mathrm{SF}=2^{i}$

Accordingly the prefix-free condition imposes a constraint on the set of spreading codes that can be assigned to active users in variable spreading CDMA systems. It is a well-known fact that the Kraft inequality determines whether a set of codes with specified lengths can be placed on the binary tree as a prefix-free set. Denoting the length of the branch from the root node $\left(C_{0,1}\right.$ in figure 1) to the $u$ th user's node by $l(u)$ and number of users by $N$, the Kraft Inequality

$$
\sum_{u=1}^{N} 2^{-l(u)} \leq 1
$$

must be satisfied to obtain a prefix-free set.

In the context of CDMA spreading codes, the Kraft inequality can be interpreted as a bandwidth constraint. Since $R_{0}$ denotes the root rate and represents the maximum achiavable total rate or the bandwidth of the system, it follows that the rate of user $u$ is

$$
R_{u}=\frac{R_{0}}{2^{l(u)}}
$$

Multiplying both sides of (1) by $R_{0}$, the Kraft inequality becomes

$$
\sum_{u=1}^{N} R_{u} \leq R_{0}
$$

which states that $R_{0}$ is an upperbound on the total data rate of all users in variable spreading CDMA case. It is trivial to generalize constraint in (2) to the Multi-Code CDMA case and $R_{0}$ represents the system bandwidth in this case.

In this paper, we consider the downlink of a single cell CDMA system. We first work on variable spreading CDMA system. We assume that we know the link gain between each user and the base station. Given the minimum rate requirements of each user and the constraint on the total BS power, our problem is to assign each user $u$ one of the OVSF codes corresponding to a data rate $R_{u}$ such that the Kraft inequality, individual data rate $\left(R_{u, \min }\right)$ and total BS power $(P)$ requirements are satisfied and the total data rate of all users (network throughput) is maximized. The problem in the Multi-Code CDMA case is similar, given the minimum rate requirements of each user and the constraint on the total BS power, we determine the number of spreading codes that will be assigned to each user such that individual data rate $\left(R_{u, \text { min }}\right)$ and total BS power $(P)$ requirements are satisfied and the network throughput is maximized.

The following section gives the formal definition of the problems, in section 3 we propose the algorithm, and in the last section prove its optimality.

\section{Problem Statements}

Problem A:

The problem formulation for variable spreading CDMA systems is as follows. Let $N$ denote the number of users in the system, $P$ denote the total BS power, $E_{b}$ denote the received energy per bit, which is assumed to be same for all users, $R_{0}$ denote the root rate, $R_{u}$ denote the assigned rate for user $u, h_{u}$ denote the link gain between user $u$ and the $\mathrm{BS}, l(u)$ denote the length of the branch from the root node $\left(C_{0,1}\right.$ in figure 1$)$ to the $u$ th user's node and $R_{u, \min }$ denote the minimum QoS requirement for user $u$, then the problem is:

$$
\begin{array}{cc}
\text { max } & \sum_{u=1}^{N} R_{u} \\
\text { subject to } & \sum_{u=1}^{N} \frac{E_{b} R_{u}}{h_{u}} \leq P \\
& \sum_{u=1}^{N} 2^{-l(u)} \leq 1 \\
& R_{u} \geq R_{u, \text { min }}, \mathrm{u}=1 . . \mathrm{N} \\
& R_{u} \in R_{\text {set }}=\left\{R_{0}, \frac{R_{0}}{2}, \frac{R_{0}}{4}, \ldots, R_{u, \text { min }}\right\}
\end{array}
$$

In the above problem formulation $E_{b} R_{u} / h_{u}$ is the transmit power required by user $u$ and (4) represents the total transmit power constraint. Note that the received energy per bit $E_{b}$ is assumed to be the same for all users, thus implying that the same coding/modulation scheme is assumed for all users. On the other hand (5) represents the bandwidth constraint, as explained in the previous section. Therefore, we consider throughput maximization under both power and bandwidth constraints.

\section{Problem B:}

The problem formulation for Multi-Code CDMA is the same except the constraints in (5) and (7). Denoting the rate corresponding to a single spreading code by $R_{\text {base }}$ and the spreading bandwidth of the system by $R_{0}$, the problem formulation in the Multi-Code case is as follows:

$\max$

$$
\begin{gathered}
\sum_{u=1}^{N} R_{u} \\
\sum_{u=1}^{N} \frac{E_{b} R_{u}}{h_{u}} \leq P \\
\sum_{u=1}^{N} R_{u} \leq R_{0} \\
R_{u} \geq R_{u, \min }, \mathrm{u}=1 . . \mathrm{N}
\end{gathered}
$$

subject to$$
R_{u} \in R_{\text {set }}=\left\{R_{\text {base }}, 2 R_{\text {base }}, 3 R_{\text {base }}, \ldots, R_{0}\right\}(12)
$$ 


\section{The Algorithms}

Before introducing the algorihtms, we first show that if the minimum required rates of users are feasible (achievable within the constraints), then the user rates in the maximum throughput-minimum power solution, among all feasible set of rates, are ordered by link gains.

Lemma 1: Given $N$ users with link gains $\bar{h}=\left[h_{1}, h_{2}, . ., h_{N}\right]$, the optimal rate assignment vector $R_{o p t}=\left[R_{1 o}, R_{2 o}, . ., R_{N_{o}}\right]$ achieving maximum throughput with minimum possible power must have the following property: the assigned rate of user $i$ is always larger than or equal to the rate of user $j, R_{i o} \geq R_{j o}$, if $h_{i}>h_{j}$.

Proof: The proof is straightforward, if $R_{i o}<R_{j o}$ and $h_{i}>h_{j}$, then we can always swap the rates of user $i$ and $j$ without changing the total sum of rates and this exchange always leads to a smaller amount of total power consumption. Denoting the initial rate assignments of $R_{i o}$ and $R_{j o}$ by $r$ and $R$, and the total power required for users $i$ and $j$ before and after swapping by $P$ and $P^{s}$ respectively, it follows that

$$
\begin{aligned}
P-P^{s} & =\left(\frac{E_{b} r}{h_{i}}+\frac{E_{b} R}{h_{j}}\right)-\left(\frac{E_{b} R}{h_{i}}+\frac{E_{b} r}{h_{j}}\right) \\
& =E_{b}(R-r)\left[\frac{1}{h_{j}}-\frac{1}{h_{i}}\right] \\
& >0
\end{aligned}
$$

(15) follows from the fact that $h_{i}>h_{j}$ and $R-r>0$.

\section{Algorithm A: The Greedy Algorithm for Variable Spreading CDMA}

In this section we introduce the greedy algorithm as a solution to the rate assignment problem, (3)-(7), in variable spreading CDMA systems. The algorithm initially attempts to provide the minimum QoS requirement of each user. If the minimum required rates are feasible, each user is assigned its corresponding minimum rate as the initial rate vector. The rest of the algorithm is greedy in nature, and the objective is to increase (to double in the binary tree case) the rate of the user who spends minimum power per bit. Since the power per bit ratio for user $u\left(P_{u} / R_{u}=E_{b} / h_{u}\right)$ is inversely proportional to the link gains $h_{u}$, the rate of the user with the highest link gain is increased first. At each greedy step, the algorithm attempts to maximize the rate of a user (users are ordered by the link gains) within the total transmit power (4) and the bandwidth constraints (5). In other words, each rate assignment is a result of a local optimum decision. In the next section we will show that the local optimum decisions lead to a global optimum solution.

We summarize the algorithm below and show how the algorithm works on the binary code tree for a sample case of 5 users in figure 2.

\section{Optimal Greedy Scheduling Algorithm for Variable Spreading CDMA}

Input: $E_{b}, R_{0}$ (root rate), $P, \bar{h}=\left[h_{1}, . ., h_{N}\right], h_{1}>h_{2}>. .>$ $h_{N}, R_{i, \min }$
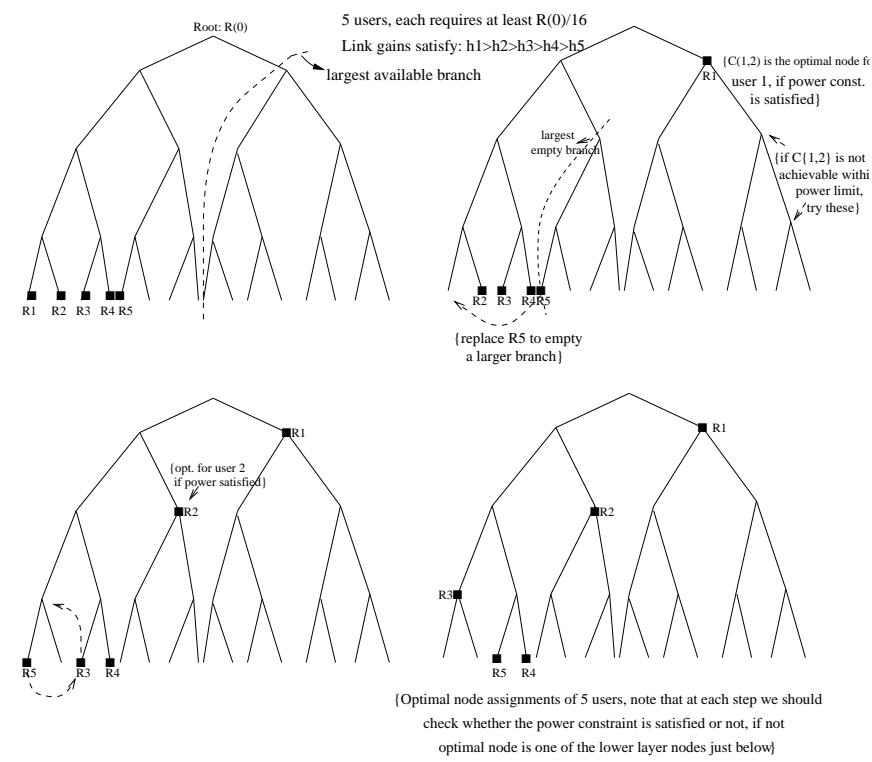

Fig. 2. Optimal Rate Scheduling Algorithm for 5 users, each requiring at least $R_{0} / 16$

Output: $\bar{R}=\left[R_{1}, \ldots, R_{N}\right]$

Initialization: $\bar{R}=\left[R_{1, \min }, \ldots, R_{N, \text { min }}\right]$, $P_{\text {total }}=\sum_{u=1}^{N} \frac{E_{b} R_{u, m i n}}{h_{u}}$

Algorithm A

for $u=1: N$

Step 1: Find the smallest positive integer $j$ satisfying

$$
\begin{gathered}
2^{-j}+\sum_{u^{\prime} \neq u}^{N} 2^{-l\left(u^{\prime}\right)} \leq 1 \\
\text { s.t } P_{\text {total }}+\frac{E_{b}\left(\frac{R_{0}}{2 j}-R_{u s e r, m i n}\right)}{h_{u s e r}}=P_{\text {total }}+P^{\prime} \leq P
\end{gathered}
$$

$P^{\prime}$ : additional power required to step up user's rate from $R_{u s e r, \min }$ to $R_{0} / 2^{j}$.

Step 2: $\bar{R}(u)=R_{0} / 2^{j}$

Step 3: $P_{\text {total }}=P_{\text {total }}+P^{\prime}$

end

An important fact is that the resulting rate assignment, $R_{0} / 2^{j}$, can be obtained by repeatedly doubling the user's initial rate as long as the constraints (4) and (5) permit.

Notice that Step 1 of Algorithm A determines the layer number $j$ of user $u$ on the binary code tree which uniquely determines the optimum rate assignment for that user $\bar{R}(u)=$ $R_{0} / 2^{j}$. However Step 1 does not tell about to which of the nodes on layer $j$ the user spreading code should be placed. On the other hand, satisfying the Kraft Inequality (16) guarantees the fact there is at least a set of $N$ spreading codes on the binary code tree such that $u$ th user's spreading code is placed on layer $j$, the rates of all other users are not affected by this placement (although spreading codes might shift on the same layers) and the set of spreading codes satisfies the prefix free property.

As an example in Figure 2, it is necessary to shift $R_{5}$ to the leftmost node in order to step up $R_{2}$ to its optimal node assignment (found by Step 1 of the algorithm) which is shown in the bottom left figure. The shifts or replacements of spreading codes on the same layer on the binary code tree is an imple- 
mentational issue and such shifts do not affect the assigned rate of a user. In this paper we are only interested in the maximum achievable network throughput and the set of rates achieving the maximum throughput. However we show that the set of optimum rates concluded by our algorithm are always realizable on the binary code tree as a result of the Kraft Inequality.

\section{Algorithm B: The Greedy Algorithm for Multi-Code CDMA}

Similar to variable spreading CDMA case, the greedy approach solves the optimal rate and spreading code assignment problem, (8)-(12), in Multi-Code CDMA systems. However, in this case the greedy rate assignment only favors the best link gain user. Namely, after the algorithm assigns the minimum required rates and allocates the correspoding spreading codes to each of the users, only the rate of the user with the best link gain is maximized using the remaining power budget.

In case there is also a maximum rate constraint on some of the users' rates, the greedy steps are executed for all users ordered by link gains.

We summarize the algorithm below.

\section{Optimal Greedy Scheduling Algorithm for Multi-Code CDMA}

Input: $E_{b}, X_{0}$ (total number of spreading codes), $P, R_{i, \min }$, $\bar{h}=\left[h_{1}, . ., h_{N}\right]$

Output: $\bar{R}=\left[R_{1}, \ldots, R_{N}\right]$ or $\bar{x}=\left[x_{1}, \ldots, x_{N}\right]$

$x_{i}$ : number of spreading codes assigned to user $\mathrm{i}$

$R_{\text {base }}$ : the rate of a single spreading code, $R_{i}=x_{i} R_{\text {base }}$

Algorithm B

Initialization: $\bar{R}=\left[R_{1, \min }, \ldots, R_{N, \min }\right]$,

$P_{\text {total }}=\sum_{u=1}^{N} \frac{E_{b} R_{u, m i n}}{h_{u}}$

for the best link gain user $u$

Step 1: Find the largest possible positive integer $x_{u}$ satisfying

$$
\begin{gathered}
x_{u}+\sum_{u^{\prime} \neq u}^{N} x_{u^{\prime}, \min } \leq X_{0} \\
\text { s.t } P_{\text {total }}+\frac{E_{b} R_{b a s e}\left(x_{u}-1\right)}{h_{u}} \leq P
\end{gathered}
$$

end

\section{Correctness And Proof of the Algorithms}

In this section we will prove the optimality of Algorithm $B$ for Multi-Code CDMA systems and the optimality of Algorithm A for Variable Spreading CDMA systems. The definition of optimality contains both maximizing the total network throughput within the constraints and also achieving the optimal throughput with minimum possible power.

The proof of the Algorithm $B$ is as follows:

Proposition 1: Algorithm B solves any instance of the combinatorial optimization problem associated with (8)(12). Moreover, if there is more than one optimal solution $\bar{R}=\left[R_{1}, R_{2}, . ., R_{N}\right]$ leading to the same optimal total network throughput, the proposed greedy scheduling algorithm yields the minimum power solution.
Proof : Let $R_{\text {base }}$ denote the rate corresponding to a single spreading code, $x_{i}$ denotes the number of spreading codes assigned to user $i, R_{i}$ denotes the rate assigned to user $i$ and $R_{i}=x_{i} R_{\text {base }}$. Therefore we scale all rate assignments by $R_{\text {base }}$ and prove the optimality of the vector $\bar{x}=\left[x_{1}, \ldots, x_{N}\right]$.

Assume the users are ordered by their link gains and user 1 is the best link gain user. Consider an optimal vector $x_{\text {opt }}$ achieving the maximum total network throughput

$$
x_{o p t}=\left[x_{1 o}, x_{2 o}, . ., x_{N o}\right]
$$

and let $x_{\text {greedy }}$ denote the vector of number of spreading codes assigned to each user after the greedy step, meaning that each user is assigned the minimum required rates and the rate of the first user is increased greedily to $x_{1, g}$

$$
x_{\text {greedy }}=\left[x_{1, g}, x_{2, \min }, . ., x_{N, \min }\right]
$$

We now rearrange the user rates in $x_{\text {opt }}(20)$ to obtain another set of rates $x_{\text {opt }}^{\prime}$ achieving the same network throughput as $x_{\text {opt }}$

$$
\begin{gathered}
x_{o p t}^{\prime}=\left[x_{1 o}^{\prime}, x_{2 o}^{\prime}, \ldots, x_{N o}^{\prime}\right] \\
x_{i o}^{\prime}=x_{i, \text { min }}, \mathrm{i}=2, \ldots, \mathrm{N} \\
x_{1 o}^{\prime}=x_{1 o}+\sum_{i=2}^{N}\left(x_{i o}-x_{i, \text { min }}\right)
\end{gathered}
$$

From $x_{o p t}$ to $x_{o p t}^{\prime}$, all user rates except the first one are reduced to the minimum required levels and the total rate reduction is assigned to the first user.

It is easy to show that $x_{o p t}^{\prime}$ requires less power than the optimal $x_{\text {opt }}$. To show this let $P_{\text {opt }}$ and $P_{\text {opt }}^{\prime}$ denote the power spent by $x_{o p t}$ and $x_{o p t}^{\prime}$ respectively, then

$$
\begin{aligned}
P_{o p t}^{\prime}-P_{o p t}= & \frac{E_{b} R_{b a s e}\left(x_{1 o}^{\prime}-x_{1 o}\right)}{h_{1}} \\
& +\sum_{u=2}^{N} \frac{E_{b} R_{b a s e}\left(x_{u o}^{\prime}-x_{u o}\right)}{h_{u}} \\
<\frac{E_{b} R_{b a s e}}{h_{1}}\left[\left(x_{1 o}^{\prime}-x_{1 o}\right)\right. & <0 \\
\left.+\sum_{u=2}^{N}\left(x_{u o}^{\prime}-x_{u o}\right)\right] & <0
\end{aligned}
$$

(26) is due to the fact that $h_{1}$ is the largest link gain and replacing $h_{u}$ by $h_{1}$ in the second expression on the right side of (25) upperbounds $P_{o p t}^{\prime}-P_{o p t}$. Also note that $x_{u o}^{\prime}-x_{u o} \leq 0$ $(u=2, \ldots, N)$ and $\left[\left(x_{1 o}^{\prime}-x_{1 o}\right)+\sum_{u=2}^{N}\left(x_{u o}^{\prime}-x_{u o}\right)\right]=0$ due to (23) and (24).

Comparing $x_{\text {opt }}$ and $x_{\text {greedy }}$, they agree on all rate assignments except the first one, (21) and (23). On the other hand for user $1, x_{1 o}^{\prime}>x_{1, g}$ can not be true since the greedy step maximizes the rate of the first user while all other users are assumed to obtain the minimum requirel levels. Moreover if $x_{1 o}^{\prime}<x_{1, g}$, then $x_{\text {greedy }}$ offers larger throughput than $x_{o p t}^{\prime}$, which achieves the same throughput as $x_{o p t}$. This can not be the case since $x_{o p t}$ is optimal by assumption and therefore it 
must offer the maximum network throughput. Thus $x_{1 o}^{\prime}=x_{1, g}$ and therefore $x_{o p t}^{\prime}=x_{\text {greedy }}$. As a result the greedy rate assignment achieves the maximum network throughput offered by $x_{o p t}$, with a smaller amount of total power consuption (26).

This concludes the proof that starting from any optimal vector $x_{o p t}$ of (20), we can obtain the same network throughput by the greedy choice $x_{\text {greedy }}$ of (21) with a smaller amount of total power consuption. The last statement also means that if there is only one optimal solution, then that is what the greedy algorithm concludes. $\square$

Proposition 2: Algorithm A solves any instance of the combinatorial optimization problem associated with (3)(7). Moreover, if there is more than one optimal solution $\bar{R}=\left[R_{1}, R_{2}, . ., R_{N}\right]$ leading to the same optimal total network throughput, the proposed greedy scheduling algorithm yields the minimum power solution.

Proof: We first start with 2 user case and prove the optimality of Algorithm $\boldsymbol{A}$ in this case. We next generalize the proof of optimality to any number of users.

\section{Step 1: The optimality for 2 user case}

A general form of the problem is as follows

$\max \quad \sum_{u=1}^{2} R_{u}$

subject to

$$
\begin{gathered}
\sum_{u=1}^{2} \frac{E_{b} R_{u}}{h_{u}} \leq P^{\prime} \\
\sum_{u=1}^{2} 2^{-l(u)} \leq \rho \\
R_{u} \geq R_{u, \min }, \mathrm{u}=1,2 \\
R_{u} \in R_{\text {set }}=\left\{R_{0}, \frac{R_{0}}{2}, \frac{R_{0}}{4}, \ldots, R_{u, \min }\right\}
\end{gathered}
$$

for any $0<P^{\prime} \leq P$ and $0<\rho \leq 1$.

Following the notation used before, let $R_{\text {opt }}$ and $R_{\text {greedy }}$ denote the vectors of the optimal and the greedy rate assignments respectively.

$$
\begin{aligned}
& R_{\text {opt }}=\left[R_{1, o p t}, R_{2, o p t}\right] \\
& R_{\text {greedy }}=\left[R_{1, g}, R_{2, g}\right]
\end{aligned}
$$

Consider the following 2 cases about $R_{1, o p t}$ and $R_{1, g}$ :

(i) $R_{1, o p t}>R_{1, g}$

The case can never occur since $R_{1, g}$ is the maximum rate that can be assigned to user 1 while user 2 is supposed to receive its minimum required data rate $R_{2, \min }$.

(ii) $R_{1, g}>R_{1, o p t}$

In this case $R_{1, \text { opt }}$ can be at most $R_{1, g} / 2$ because of the geometric relationship between the rates on the binary code tree. On the other hand, by Lemma $1, R_{1, o p t}$ is always larger than or equal to $R_{2, o p t}$, therefore the sum of $R_{1, o p t}$ and $R_{2, o p t}$ can never exceed the sum of $R_{1, g}$ and $R_{2, g}$, since $R_{2, g}$ is nonzero

$$
\begin{aligned}
R_{2, o p t} \leq & R_{1, o p t} \leq R_{1, g} / 2 \Longrightarrow R_{2, o p t}+R_{1, o p t} \leq R_{1, g} \\
& \Longrightarrow R_{2, o p t}+R_{1, o p t}<R_{1, g}+R_{2, g}
\end{aligned}
$$

Therefore case(ii) can never occur since $R_{\text {opt }}$ should have led to the maximum network throughput. As a result, $R_{1, o p t}=R_{1, g}$.
Since $R_{2, g}$ is the maximum rate that can be assigned to user 2 within the constraints and assuming the first user is assigned $R_{1, g}, R_{2, o p t}$ should also be equal to $R_{2, g}, R_{2, g}=R_{2, o p t}$. It is clear that the greedy rate assignment vector is also the minimum power solution. The argument behind this fact is similar to the argument in the proof of Lemma 1: Among the set of rate assignments $\bar{R}=\left[R_{1}, R_{2}\right]$ leading to the same maximum network throughput, the one which assigns the highest possible rate to the user with better link gain is the minimum power solution, since that user consumes less power per bit.

\section{Step 2: The optimality in general}

Assume that there are $N$ users in the system and the greedy algorithm is the optimal way to assign the rates of those $N$ users. Assuming this, the next question is whether the greedy algorithm is optimal for $N+1$ users as well, if it is assumed to be optimal for $N$ users.

Let $R_{\text {greedy }}$ and $R_{\text {opt }}$ denote the greedy rate assignments and the optimal rate assignments respectively for $N+1$ users. The users are ordered such that $(N+1)$ th user is the worst link gain user and cannot receive a larger rate than any of the other $N$ users by Lemma 1.

$$
\begin{gathered}
R_{\text {greedy }}=\left[R_{1, g}, R_{2, g}, . ., R_{N, g}, R_{N+1, g}\right] \\
R_{\text {opt }}=\left[R_{1, o p t}, R_{2, o p t}, . ., R_{N, o p t}, R_{N+1, o p t}\right]
\end{gathered}
$$

A preliminary fact that will be used throughout the proof is that due to the $N$ user optimality assumption of the greedy algorithm, if we assume to fix the rate of the $(N+1)$ th user to $R_{N+1, \text { opt }}$ and determine the rest of $N$ rate assignments greedily, the resulting set of rates must be optimal. In other words the first $N$ rate assignments of $R_{\text {opt }}$ can be obtained by the greedy solution of the following problem:

$$
\begin{array}{rc}
\max & \sum_{u=1}^{N} R_{u}\left(R_{N+1}=R_{N+1, o p t}\right) \\
\text { subject to } & \sum_{u=1}^{N} \frac{E_{b} R_{u}}{h_{u}} \leq P-\frac{E_{b} R_{N+1, o p t}}{h_{N+1}} \\
& \sum_{u=1}^{N} 2^{-l(u)} \leq 1-R_{N+1, o p t} / R_{0} \\
& R_{u} \geq R_{u, \min }, \mathrm{u}=1 . . \mathrm{N} \\
& R_{u} \in R_{\text {set }}=\left\{R_{0}, \frac{R_{0}}{2}, \frac{R_{0}}{4}, \ldots, R_{u, \text { min }}\right\}
\end{array}
$$

Notice that in Step 1, we proved the greedy optimality for $N=$ 2 and for any $0<P^{\prime} \leq P$ and $0<\rho \leq 1$, (27)-(31).

The rest of the proof shows that either $R_{\text {greedy }}$ is optimal or there is no feasible $R_{o p t}$. To show this fact we compare the relative powers spent by $R_{\text {greedy }}$ and $R_{\text {opt }}$ denoted by $P^{g}$ and $P^{o}$ respectively.

Consider the rate assignments by (36) and (37). Notice that, from user 1 to user $N+1$, for the first user for which $R_{\text {greedy }}$ and $R_{o p t}$ differ, the optimal assignment must be lower than the greedy assignment. We name the index of this user as $u_{1}\left(u_{1} \geq\right.$ 1), thus

$$
\begin{gathered}
R_{i, g}=R_{i, o p t}, \mathrm{i}=1, \ldots, u_{1}-1 \\
R_{u_{1}, g}>R_{u_{1}, o p t}
\end{gathered}
$$

The reasoning behind (44) is that the greedy algorithm locally maximizes the rate of a user at hand starting from the best link 
gain user, therefore if $R_{u_{1}, \text { opt }}>R_{u_{1}, g}$ were true, then $R_{u_{1}, g}$ would not be the locally maximum choice for the $u_{1}$ th user due to (44).

Similar to $u_{1}$, we name the index of those other users, for which the optimal assignment determines a lower rate than the greedy assignment, by $u_{2}, \ldots, u_{M}(M \leq N)$. Thus

$$
\begin{gathered}
R_{i, g}>R_{i, o p t}, \mathrm{i}=u_{1}, u_{2}, \ldots, u_{M} \\
R_{j, g}=R_{j, o p t} \text { or } R_{j, g}<R_{j, o p t}, j \neq i, j \leq N
\end{gathered}
$$

The difference in power spent by the optimal and the greedy rate assignments for users $u_{1}, \ldots, u_{2}-1$ is denoted by $P_{\text {diff, }, 1}$, similary the difference in sum of rates for the same users is denoted by $R_{\text {diff }, 1}$

$$
\begin{array}{r}
R_{\text {diff }, 1}=\sum_{i=u_{1}}^{u_{2}-1}\left(R_{i, o p t}-R_{i, g}\right) \\
P_{d i f f, 1}=\frac{E_{b}\left(R_{u_{1}, o p t}-R_{u_{1}, g}\right)}{h_{u_{1}}}+\sum_{i=u_{1}+1}^{u_{2}-1} \frac{E_{b}\left(R_{i, o p t}-R_{i, g}\right.}{h_{i}}(48) \\
P_{\text {diff }, 1}>\frac{E_{b}}{h_{u_{1}}} \sum_{i=u_{1}}^{u_{2}-1}\left(R_{i, o p t}-R_{i, g}\right)=\frac{E_{b} R_{d i f f, 1}}{h_{u_{1}}}
\end{array}
$$

The last inequality follows from the fact that $h_{u_{1}}>h_{i}$ for each $i$ in (48) and therefore replacing $h_{i}$ by $h_{u_{1}}$ in (48) lowerbounds $P_{\text {diff }, 1}$.

An important observation on (47)-(49) is that $R_{\text {diff }, 1}$ is nonpositive. Before explaining why it is so, remember that due to $N$ user optimality assumption of the greedy algorithm, the first $N$ rate assignments of $R_{o p t}$ can be obtained as the greedy solution of (38)-(42) assuming $(N+1)$ th user's rate is fixed to $R_{N+1, \text { opt }}$, thus in fact $R_{u_{1}, \text { opt }}$ is a locally optimum decision if the original $N+1$ user problem is reduced to an $N$ user problem by the assumption on $(N+1)$ th user's rate. Now consider the case where $R_{\text {diff }, 1}$ is positive. Then $P_{\text {diff }, 1}$ is also positive due to (49), which implies that $R_{\text {opt }}$ remains feasible if $\left[R_{u_{1}, o p t}, \ldots, R_{u_{2}-1, o p t}\right]$ is replaced by $\left[R_{u_{1}, g}, \ldots, R_{u_{2}-1, g}\right]$ and the rest of user rates remain the same in $R_{o p t}$. In this case the locally maximum choice of a greedy algorithm at $u_{1}$ th step (greedy assumes minimum rates for successive users) can not be $R_{u_{1}, o p t}$ due to (44). The contradiction leads us to the point that $R_{\text {dif } f, 1}$ is not positive.

Similar to $R_{\text {diff,1 }}$ and $P_{\text {diff }, 1}$, the difference in power spent by the optimal and the greedy rate assignments for users $u_{j}, \ldots, u_{j+1}-1$ is denoted by $P_{\text {diff }, j}$ and the difference in sum of rates for the same users is denoted by $R_{d i f f, j}$. The lower bound on $P_{\text {diff }, 1}$ and the nonpositivity of $R_{\text {diff }, 1}$ extend to $R_{d i f f, j}$ and $P_{d i f f, j}$ as well. Remember that there are $M$ users, among the first $N$ users, for which the optimal assignnment is lower than the greedy assignment (45).

$$
\begin{gathered}
R_{\text {diff }, j}=\sum_{i=u_{j}}^{u_{j+1}-1}\left(R_{i, o p t}-R_{i, g}\right) \leq 0 \\
P_{d i f f, j}>\frac{E_{b} R_{d i f f, j}}{h_{u_{j}}}, \mathrm{j}=1, \ldots, \mathrm{M}-1 \\
R_{\text {diff }, M}=\sum_{i=u_{M}}^{N}\left(R_{i, o p t}-R_{i, g}\right) \leq 0 \\
P_{\text {diff }, M}>\frac{E_{b} R_{d i f f, M}}{h_{u_{M}}}, \mathrm{j}=\mathrm{M}
\end{gathered}
$$

Let $P^{g}$ and $P^{o}$ denote the total power spent by $R_{\text {greedy }}$ and $R_{o p t}, R^{g}$ and $R^{o}$ denote the total sum of rates of $R_{\text {greedy }}$ and $R_{\text {opt }}$ respectively, using (50)-(53)

$$
\begin{gathered}
R^{o}-R^{g}=\sum_{u=1}^{N+1}\left(R_{u, o p t}-R_{u, g}\right) \\
=\sum_{j=1}^{M} R_{d i f f, j}+\left(R_{N+1, o p t}-R_{N+1, g}\right) \\
P^{o}-P^{g}=\sum_{u=1}^{N+1} \frac{E_{b}\left(R_{u, o p t}-R_{u, g}\right)}{h_{u}} \\
=\sum_{j=1}^{M} P_{d i f f, j}+\frac{E_{b}}{h_{N+1}}\left(R_{N+1, o p t}-R_{N+1, g}\right) \\
P^{o}-P^{g}>\sum_{j=1}^{M} \frac{E_{b} R_{d i f f, j}}{h_{u_{j}}}+\frac{E_{b}}{h_{N+1}}\left(R_{N+1, o p t}-R_{N+1, g}\right)
\end{gathered}
$$

Since $h_{N+1}$ is the smallest link gain, $R_{\text {diff }, j}$ is nonpositive for each $j$ due to (50) and using (54), we can further lowerbound (56) as follows

$$
\begin{gathered}
P^{o}-P^{g}>\sum_{j=1}^{M} \frac{E_{b} R_{d i f f, j}}{h_{N+1}}+\frac{E_{b}}{h_{N+1}}\left(R_{N+1, o p t}-R_{N+1, g}\right) \\
=\frac{E_{b}}{h_{N+1}}\left(R^{o}-R^{g}\right)
\end{gathered}
$$

Eq (57) has a nice interpretation, among the set of rates leading to the same optimal network throughput $\left(R^{o}-R^{g}=0\right)$, the greedy one minimizes the total power $\left(P^{o}-P^{g}>0\right)$. We will now show that if there is any feasible $R_{o p t}$, then $R_{\text {greedy }}$ always achieves the maximum network througput offered by $R_{o p t}$ and since it also minimizes the total power, it is in fact the optimal one.

Consider (54), in order for $R_{o p t}$ to be optimal, it should have the maximum sum of rates, thus

$$
\begin{gathered}
R^{o}-R^{g}=R_{N+1, o p t}-R_{N+1, g}+\sum_{j=1}^{M} R_{d i f f, j} \geq 0 \\
R_{N+1, o p t}-R_{N+1, g} \geq 0
\end{gathered}
$$

(59) follows from the fact that $R_{d i f f, j}$ is nonpositive for each $j$ due to (50). By Lemma 1 the user rates are ordered by the link gains, using this fact and (59), it follows that $R_{N+1, g}$ is the smallest rate assignment of all $R_{u, g}$ and $R_{u, o p t}$. Therefore, using the geometric relationship between the rates on the binary code tree, all rate assignments by $R_{\text {greedy }}$ and $R_{o p t}$ are expressible in terms of integer multiples of $R_{N+1, g}$

$$
R_{u, o p t}, R_{u, g}=a R_{N+1, g}, a=2^{k}, k \geq 0
$$

In this case the difference in throughput offered by $R_{\text {greedy }}$ and $R_{o p t}$ can also be expressed as an integer multiple of $R_{N+1, g}$

$$
R^{o}-R^{g}=\sum_{u=1}^{N+1}\left(R_{u, o p t}-R_{u, g}\right)=j R_{N+1, g}, j \geq 0
$$


Consider $R_{\text {greedy }}$ (36). At $(N+1)$ th greedy step, the maximum rate that can be assigned to the $(N+1)$ th user within available resources is $R_{N+1, g}$. At this point 2 case might have occured: Case-1) the system is power limited and although there is available bandwidth, $(N+1)$ th user cannot receive $2 R_{N+1, g}$ because of the power constraint. Case-2) the system is bandwidthlimited and although there is available power, $(N+1)$ th user cannot receive $2 R_{N+1, g}$ because most of the bandwidth has already been used.

For Case-1, the power required to assign an additional $R_{N+1, g}$ to the $(N+1)$ th user is less than the residual power of $R_{\text {greedy }}$. Denoting the total available power by $P$ and rewriting the lowebound (57) as an equality

$$
\begin{gathered}
P-P^{g}<\frac{E_{b} R_{N+1, g}}{h_{N+1}} \\
\left.P^{o}-P^{g}=\frac{E_{b}}{h_{N+1}}\left(R^{o}-R^{g}\right)+\epsilon, \text { for an arbitrary } \epsilon>\emptyset 63\right)
\end{gathered}
$$

Combining (62) and (63) we obtain a relationship between the total power spent by the optimal assignments $P^{o}$ and the total available power $P$, then using (61) we come up with the final inequality (65)

$$
\begin{gathered}
P-P^{o}<\frac{E_{b} R_{N+1, g}}{h_{N+1}}-\frac{E_{b}}{h_{N+1}}\left(R^{o}-R^{g}\right)-\epsilon \\
P-P^{o}<(1-j) \frac{E_{b} R_{N+1}}{h_{N+1}}-\epsilon
\end{gathered}
$$

for a real arbitrary $\epsilon>0$ and for an integer $j \geq 0$. In order for $R_{\text {opt }}$ to be feasible, it should satisfy the following inequality

$$
P-P^{o} \geq 0
$$

Thus according to (65), $R_{o p t}$ is feasible only if $j=0$. In this case, from (61), the optimal and the greedy rate assignments offer the same total network throughput

$$
R^{o}=R^{g}
$$

For Case-2, denoting the total available bandwidth by $R_{0}$, the total sum of rates of $R_{\text {greedy }}$ by $R^{g}$, the remaining bandwidth out of $R^{g}$ is less than $R_{N+1, g}$ so that $(N+1)$ th user can only get $R_{N+1, g}$ instead of $2 R_{N+1, g}$ although there is available power. Using (61)

$$
\begin{gathered}
R_{0}-R^{g}<R_{N+1, g} \\
R^{o}-R^{g}=j R_{N+1, g}, j \geq 0 \\
R_{0}-R^{o}<R_{N+1, g}-j R_{N+1, g}
\end{gathered}
$$

The total sum of rates offered by $R_{o p t}$ can not exceed the total available bandwidth $R_{0}-R^{o} \geq 0$. In this case, $R_{o p t}$ is only achievable if $j=0$. In this case, from (61), the optimal and the greedy rate assignments offer the same total network throughput (67).

To sum up, we started with the assumption that the greedy algorithm is the optimal way to assign the rates of $N$ users. Using this assumption, we have shown that the greedy algorithm achieves the maximum network throughput for $N+1$ user as well. Moreover (57) implied that among the set of rates leading to the same optimal network throughput $\left(R^{o}-R^{g}=0\right)$, the greedy one minimizes the total power $\left(P^{o}-P^{g}>0\right)$, thus proving that the greedy rate assignment is in fact the optimal one for $N+1$ user. The proof of optimality of the greedy algorithm for 2 user case is therefore generalized to any number of users by induction. $\square$

\section{CONCLUSION}

\section{REFERENCES}

[1] F.Adachi,M.Sawahashi and H.Suda, "Wideband DS-CDMA for NextGeneration Mobile Communication Systems", IEEE Communications Magazine, Volume: 36, Issue: 9, Sept. 1998, Page(s): 56 -69.

[2] F.Adachi,M.Sawahashi, and K.Okawa, "Tree-structured generation of orthogonal spreading codes with different lengths for forward link of DSCDMA mobile radio", IEEE Electronics Letters, Volume: 33, Issue: 1,Jan. 1997, Page(s): $27-28$.

[3] Thit Minn; Kai-Yeung Siu, "Dynamic assignment of orthogonal variablespreading-factor codes in W-CDMA" IEEE Journal on Selected Areas in Communications, Volume: 18, Issue: 8, Aug. 2000, Page(s): 1429 -1440

[4] Kam, A.C.; Minn, T.; Siu, K.-Y, "Supporting rate guarantee and fair access for bursty data traffic in W-CDMA" IEEE Journal on Selected Areas in Communications, Volume: 19, Issue: 11, Nov. 2001, Page(s): 2121 -2130

[5] Yu-Chee Tseng; Chih-Min Chao; Shih-Lin Wu, "Code placement and replacement strategies for wideband CDMA OVSF code tree management" IEEE Global Telecommunications Conference, 2001. GLOBECOM '01, Page(s): 562 -566 vol.1

[6] Dell'Amico, M.; Merani, M.L.; Maffioli, F., "Efficient algorithms for the assignment of OVSF codes in wideband CDMA", IEEE International Conference on Communications, 2002. ICC 2002., Volume: 5, Page(s): 3055 $-3060$ 